\title{
Délimitation du système urbain quotidien
}

Delimitation of the daily urban system

Abgrenzung des täglischen Stadtsystems

\section{Christophe Enaux}

\section{OpenEdition}

\section{Journals}

Édition électronique

URL : http://journals.openedition.org/rge/4380

DOI : $10.4000 /$ rge.4380

ISSN : 2108-6478

\section{Éditeur}

Association des géographes de l'Est

Édition imprimée

Date de publication : 1 avril 1999

ISSN : 0035-3213

\section{Référence électronique}

Christophe Enaux, « Délimitation du système urbain quotidien », Revue Géographique de l'Est [En ligne], vol. 39 / 2-3 | 1999, mis en ligne le 04 septembre 2013, consulté le 08 septembre 2020. URL : http:// journals.openedition.org/rge/4380 ; DOI : https://doi.org/10.4000/rge.4380

Ce document a été généré automatiquement le 8 septembre 2020.

Tous droits réservés 


\title{
Délimitation du système urbain quotidien
}

\author{
Delimitation of the daily urban system \\ Abgrenzung des täglischen Stadtsystems
}

\section{Christophe Enaux}

1 Parmi les objets géographiques, la ville en est un des plus complexes à tel point qu'il est difficile d'en donner une définition qui tienne compte de l'ensemble de ses dimensions. La ville est généralement prise comme un lieu qui permet des économies d'agglomération, fondées sur la proximité des externalités produites par les acteurs. Ces idées de relations, d'interrelations, sont exprimées explicitement dans la définition de P. Claval (1984) : « la ville est une machine à maximiser les interactions sociales ». Du point de vue d'une représentation concrète, elle correspond pour certains à une morphologie particulière basée sur la continuité d'un grand nombre de constructions, intégrant une certaine quantité de population. D'autres insistent sur le caractère des activités et des fonctions non agricoles pour la définir. D'autres encore privilégient le mode de vie des habitants ou incluent dans la ville toutes les personnes qui la fréquentent couramment pour se rendre à leur lieu de travail, même si leur habitation est située en dehors.

2 La pluralité des acceptions de l'objet ville peut être synthétisée par l'intermédiaire de la notion de système urbain qui présente l'avantage de mettre l'accent sur les composantes urbaines et leurs caractéristiques et relations, et d'autoriser une délimitation spatiale du système selon l'objectif poursuivi. Dans le cas présent, nous nous attacherons à la délimitation du système urbain quotidien, c'est-à-dire du système spatial intégrant les lieux d'activité de la vie journalière des individus. Comment délimiter un tel système, la façon de le mesurer concrètement, constituent les éléments développés ci-dessous. 


\section{Logique de délimitation}

3 La notion même de système fait référence aux relations entre différentes parties. La délimitation du système urbain quotidien est par conséquent un phénomène qui s'appuie sur les liens entre des lieux de l'espace géographique, au détriment des limites directement observables dans ce dernier. L'aire urbaine retenue par l'I.N.S.E.E. (T. Le Jeannic, 1996) correspond à cette logique tout en privilégiant une approche centre/ périphérie. A partir d'un cœur urbain défini a priori, des lieux sont rattachées à celui-ci de façon itérative selon qu'ils possèdent des relations supérieures à un seuil prédéterminé avec l'ensemble en cours de constitution. Ce principe de construction de l'aire urbaine est semblable à celui mis en œuvre pour les Standard Metropolitan Statistical Areas (S.M.S.A.) des Etats-Unis (B. J. L. Berry et F. E. Horton, 1970) ou des Standard Metropolitan Labour Areas (S.M.L.A.). de Grande-Bretagne (D. Pumain et al., 1990).

4 Une alternative à cette première approche qui nécessite la définition d'un seuil de relation a priori dont la valeur possède une forte probabilité de changer de signification au cours du temps, est de considérer les relations entre les lieux sans tenir compte de leur orientation. S'il existe des liens unilatéraux ou réciproques privilégiés entre des lieux, alors des regroupements de lieux sont identifiables, sans que l'on ait à établir des seuils préalables de liens, en se fondant uniquement sur les discontinuités d'intensité de relations entre différents ensembles de lieux. L'unique contrainte a posteriori pour qualifier le système spatial résultant de système urbain, est qu'il contienne une unité urbaine selon l'acceptation de l'I.N.S.E.E.

5 La logique de délimitation d'un système urbain quotidien pose donc le problème du choix de la relation formant le ciment du système spatial. Les motifs de déplacement vers les lieux d'activité de la vie journalière des individus sont nombreux et variés. Les plus fréquents restent cependant les déplacements de travail, scolaires, d'achats et de loisirs, encore que ces deux derniers types sont de plus en plus souvent combinés, chez les actifs ayant un emploi, avec le trajet aller/retour entre le domicile et le travail ou s'effectuent lors de la pause de midi (J.-P. Orfeuil et al., 1989). Si l'on réunit les plus fortes fréquences et la distance des déplacements, il est clair que ce sont les distances des déplacements de travail qui correspondent aux plus grands écarts entre les lieux d'activités de la vie journalière d'autant, que ce sont généralement les personnes ayant un emploi qui disposent du budget espace (C. Marchetti, 1991) quotidien le plus important.

6 Ainsi les déplacements de travail peuvent-ils constituer la variable de relation permettant de déterminer les lieux constitutifs du système urbain quotidien. La délimitation d'un système urbain quotidien revient donc à identifier dans un ensemble $n$ de lieux, les sous-ensembles $i$ basés sur les discontinuités de relations de déplacements de travail.

\section{Principe méthodologique de délimitation}

7 Les déplacements de travail sont relevés en France par le biais du recensement général de la population. Ils sont fournis par l'I.N.S.E.E. à l'échelle communale, formant ainsi des flux de déplacements qui matérialisent le nombre de personnes résidant dans une commune et allant travailler dans une autre ou des quantités d'individus habitant et 
exerçant leur activité professionnelle dans la même commune. Ces quantités peuvent être regroupées dans un tableau géographique d'échanges comprenant respectivement en lignes et en colonnes, les communes de résidence et de travail (fig. 1). Ce tableau doit répondre à deux contraintes initiales.

Figure 1 : Tableau d'échanges des déplacements de travail

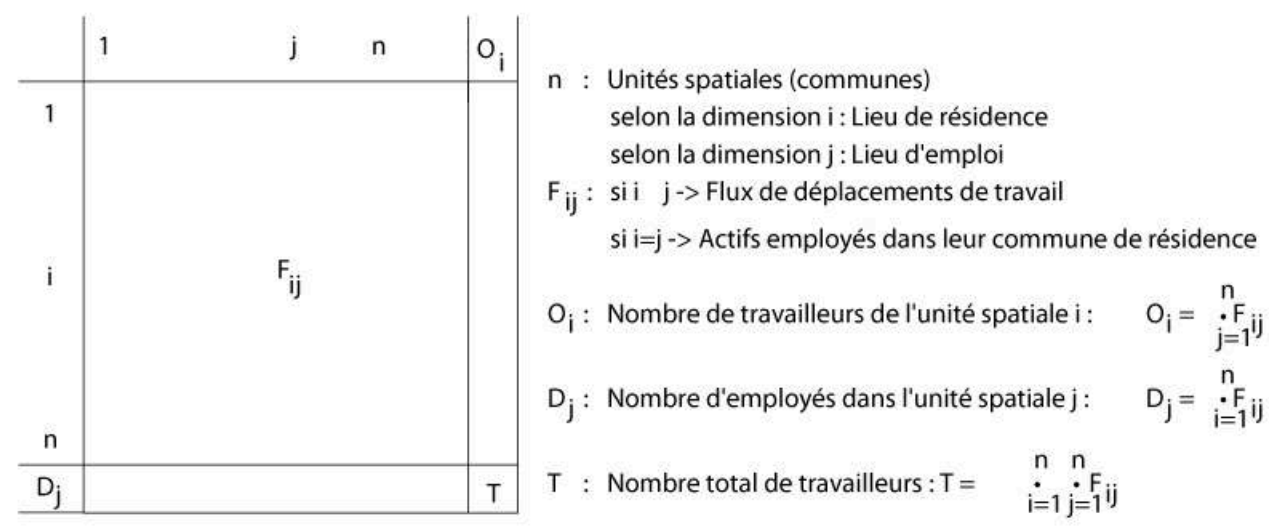

Auteur: C. ENAUX

\section{A. Contraintes sur le tableau d'échanges}

Les ensembles de lieux présentant des relations privilégiées de flux de déplacements de travail possèdent une extension spatiale qui n'est pas connue a priori. Ce phénomène implique que le tableau couvre une portion d'espace géographique suffisamment vaste pour contenir des systèmes urbains quotidiens. Dans le cas contraire, ces derniers seront tronqués sur leur périphérie ou pourront contenir des communes ayant des relations privilégiées avec des lieux situés en dehors de l'espace représenté par le tableau. Couvrir une zone géographique suffisamment vaste et maintenir l'ouverture du tableau d'échanges en prenant en compte les relations des lieux du tableau avec ceux formant l'environnement de ce dernier, sont deux conditions initiales indispensables à la cohérence des systèmes urbains quotidiens obtenus.

Prises telles quelles, les quantités contenues dans le tableau traduisent des masses de relation entre les communes. Si l'on se base sur celles-ci pour déterminer les communes ayant des relations privilégiées, une analyse fondée sur ces masses va favoriser le regroupement de communes les plus importantes au détriment des autres. Pour mettre en évidence la structure relationnelle unissant l'ensemble des communes, une transformation des déplacements de travail permet de faire apparaitre l'intensité pondérée de relations.

\section{B. Mesure de l'intensité pondérée de relations}

10 Les formulations mesurant l'intensité pondérée de relations entre des lieux sont nombreuses. L'exemple le plus connu permettant de construire des groupes d'unités spatiales présentant des échanges privilégiés correspond au lien Intramax (I. Masser et P. J. B. Brown, 1975). 


\section{Lien Intramax}

11 Ce lien reprend dans les grandes lignes les travaux de J.H. Ward et peut s'écrire sous la forme suivante :

$$
\begin{aligned}
& \operatorname{Intramax}_{\mathrm{i}-\mathrm{j}}=\frac{\mathrm{F}_{\mathrm{ij}}}{\mathrm{O}_{\mathrm{i}} \cdot \mathrm{D}_{\mathrm{j}}}+\frac{\mathrm{F}_{\mathrm{ij}}}{\mathrm{O}_{\mathrm{j}} \cdot \mathrm{D}_{\mathrm{j}}} \rightarrow \text { maximum } \\
& \text { sachant que: } \forall \text { i et } \mathrm{j}, \mathrm{O}_{\mathrm{i}}>0 \text { et } \mathrm{D}_{\mathrm{j}}>0 .
\end{aligned}
$$

Pour comprendre ce qu'exprime ce lien, il suffit de le reformuler différemment. En le multipliant par une constante quelconque a supérieure à zéro, on obtient :

$$
\left.\operatorname{Intramax}_{i-j}=a \cdot \mid \frac{F_{i j}}{O_{i} \cdot D_{j}}+\frac{F_{j i}}{O_{j} \cdot D_{i}}\right\rfloor \rightarrow \text { maximum }
$$

Comme a $\cdot \frac{b}{c}=\frac{b}{\frac{c}{a}}$, on a alors :

$$
\text { Intramax }_{i-j}=\frac{F_{i j}}{\frac{0_{i} \cdot D_{j}}{a}}+\frac{F_{j i}}{0_{j} \cdot D Z}+\text { maximum }
$$

Il est possible de choisir la constante a égale à $\mathrm{T}$ qui représente le nombre total de déplacements entre les lieux du tableau d'échanges ou celui des travailleurs. Les éléments $\left(\mathrm{O}_{\mathrm{i}} \cdot \mathrm{D}_{\mathrm{j}}\right) / \mathrm{a}$ et $\left(\mathrm{O}_{\mathrm{j}} \cdot \mathrm{D}_{\mathrm{i}}\right) / \mathrm{a}$ matérialisent alors des flux théoriques entre deux lieux sous l'hypothèse d'indépendance statistique (encore appelée hypothèse nulle) de la population active au lieu de travail (D) et de celle au lieu de résidence (O). Cette hypothèse stipule qu'il n'existe aucun lien entre ces deux types de population dans un échantillon de travailleurs de quantité T. Ceci revient à dire que la distribution de la totalité des travailleurs $\mathrm{T}$ dans les cellules d'une matrice de flux théoriques est uniquement fonction de la capacité marginale d'un lieu à émettre de la population active $\left(\mathrm{O}_{\mathrm{i}}\right)$ ou à accueillir de la population active $\left(\mathrm{D}_{\mathrm{j}}\right)$. L'idée sous-jacente à ce lien est donc que deux lieux entretiennent de fortes relations si le rapport entre le flux observé et le flux théorique est maximum.

Ce lien Intramax favorise cependant le regroupement prioritaire des lieux dont la population active est la plus faible. L'exemple suivant illustre cette propriété :

- Lien $i-j=5 /(10 * 15)+10 /(20 * 30)=0.05$

- Lien $k-l=10 /(20 * 30)+20 /(40 * 60)=0.025$

Pour quatre lieux $\mathrm{i}, \mathrm{j}, \mathrm{k}$ et $\mathrm{l}$ au sein d'un tableau d'échanges entretenant deux à deux $\mathrm{i}-\mathrm{j}$ et k-l les mêmes proportions de flux, mais dont les quantités de flux échangées et totales sont respectivement deux fois plus importantes, le lien associe prioritairement les unités $\mathrm{i}$ et $\mathrm{j}$ de plus faibles populations de travailleurs, au lieu de résidence ou au lieu d'emploi, en raison de l'opération de multiplication. Ce fait est le problème principal de cette définition de lien. En privilégiant le regroupement des unités à faible 
population, les plus grandes ne sont qu'ajoutées à de vastes sous-ensembles de lieux indiquant qu'elles ont des liens avec de nombreuses unités, mais sans faire apparaitre les sous-ensembles restreints dont elles font partie. Cette caractéristique n'est pas fondamentale lorsque l'on identifie l'extension maximale d'un système urbain. Mais elle pose un problème dans toutes les situations intermédiaires où l'on recherche avant tout des zones de fortes cohésions économiques et sociales comme les systèmes urbains quotidiens. Une autre mesure de l'intensité pondérée de relations a donc été définie.

\section{Lien du degré d'interaction}

Le lien du degré d'interaction traduit qu'un lieu i possède une importante relation avec un lieu j, si i envoie, par rapport à sa capacité d'émission $\left(\mathrm{O}_{\mathrm{i}}\right)$, une forte proportion de personnes vers $\mathrm{j}$, mais également si ce nombre de personnes se rendant vers $\mathrm{j}$ représente une forte proportion de la totalité des actifs au lieu d'emploi du lieu $\mathrm{j}\left(\mathrm{D}_{\mathrm{j}}\right)$. Comme ce lien est fondé sur les deux sens de l'interaction entre $\mathrm{i}$ et $\mathrm{j}$, il est formé de quatre rapports additionnés les uns aux autres :

Degré d'interaction $n_{i-j}=\frac{F_{i j}}{O_{i}}+\frac{F_{i j}}{D_{j}}+\frac{F_{j i}}{O_{j}}+\frac{F_{j i}}{D_{i}} \rightarrow$ maximum (4)

Le lien ainsi défini varie entre 0 et 4 , et n'est valable que dans les situations où les capacités totales d'émission et de réception d'un lieu sont supérieures à zéro. Il peut arriver que celles-ci soient nulles; dans ce cas, le lieu ne fait partie d'aucun sousensemble de lieux fondé sur les flux de déplacements de travail. Lorsque la capacité $\mathrm{O}_{\mathrm{i}}$ ou $\mathrm{D}_{\mathrm{i}}$ est égale à zéro, cela signifie qu'une unité spatiale ne reçoit ou n'émet aucun flux. Plutôt que de l'exclure a priori d'un sous-ensemble de lieux auquel elle participe uniquement comme unité résidentielle ou d'emploi, de la rattacher à une autre unité, ce qui modifie à la fois la répartition des flux et leur signification, nous admettrons des définitions complémentaires du lien du degré d'interaction. S'il unit un lieu i ne possédant qu'une population active résidentielle avec un autre $\mathrm{j}$ n'ayant qu'une population active au lieu d'emploi, le degré d'interaction s'exprime unilatéralement sous la forme :

$$
\text { Degré d'interaction } n_{i-j}=\frac{F_{i j}}{O_{i}}+\frac{F_{i j}}{D_{j}} \rightarrow \text { maximum }
$$

Il se peut également que le lien porte sur deux unités spatiales au profil identique, c'est-à-dire ne possédant, par exemple, qu'une population active au lieu de résidence. Ce phénomène traduit une situation dans laquelle il ne peut $\mathrm{y}$ avoir de flux entre les unités. Le lien de degré d'interaction représente alors une absence de liaison. Ce lien prend ainsi en compte tous les cas de figure observables d'unités spatiales. Il recouvre des situations différentes dans lesquelles des relations entre des lieux existent ou non, que ces relations soient unilatérales ou qu'elles s'effectuent dans les deux sens. Le lien du degré d'interaction paraît être adapté à la recherche de groupes de lieux entretenant des liaisons privilégiées puisqu'il embrasse l'ensemble des types de relation et leur intensité, tout en garantissant un poids identique des lieux. L'intensité pondérée de relations est associée à 
une stratégie de regroupement des lieux afin de déterminer le système urbain quotidien.

\section{Stratégie de regroupement des individus}

19 Les liens privilégiés de flux de déplacements de travail entre certaines unités spatiales n'empêchent pas que ces sous-ensembles possèdent également des relations avec d'autres groupes. L'agencement ordonné de l'ensemble de ces relations forme une structure d'emboîtement hiérarchique qui peut être mise en évidence par une classification ascendante hiérarchique. Elle nécessite que l'on dispose d'une mesure de la ressemblance entre les individus et d'un critère permettant de les regrouper.

\section{Mesure de la ressemblance des lieux}

La mesure de ressemblance entre les individus est effectuée à l'aide d'une distance dont les plus courantes sont la distance euclidienne et celle du khi-deux. Lorsqu'elle est nulle, les individus sont identiques. Plus cette distance s'accroît et tend vers l'infini, plus les différences entre ceux-ci sont grandes. Il arrive fréquemment, notamment dans les sciences sociales, que l'on ne dispose pas à proprement parler d'un tableau de distances entre des individus. Le tableau correspond plutôt à une matrice de proximités dont le contenu est également quantifié et symétrique par rapport à la diagonale qui, elle-même, est égale à zéro. Les différences entre proximité et distance proviennent essentiellement de l'échelle de variation des valeurs et des propriétés mathématiques associées aux distances.

21 Le lien du degré d'interaction présenté au point précédent peut se ramener à une telle matrice de proximités. Plus le lien entre deux unités sera fort, plus l'écart qui les sépare sera réduit. Concrètement, pour obtenir cette proximité des lieux, il suffit de prendre le complémentaire du lien du degré d'interaction entre deux unités. Sachant que ce degré d'interaction est au maximum de 4 , la proximité entre deux lieux $i$ et $\mathrm{j}$ est alors égale à ce maximum moins le lien du degré d'interaction entre $i$ et $j$. Lorsque $i$ et $j$ sont identiques, l'écart est par principe nul.

L'examen des propriétés du tableau des proximités permet de définir la famille de distances auquel il appartient. La distance euclidienne se distingue des autres types de distance par les quatre propriétés qui lui sont associées (non négativité, symétrie, identité, inégalité triangulaire). Le tableau des proximités issu du complémentaire du lien du degré d'interaction, satisfait la condition de non négativité. En effet, le lien i-j étant au maximum de quatre, la distance calculée à l'aide du complémentaire ne peut être inférieure à zéro. Cette proximité est symétrique car le lien du degré d'interaction est fondé sur la liaison de $i$ vers $j$ et de $j$ vers $i$. Par contre, elle ne vérifie pas la condition d'identité : si la distance entre deux points est nulle, ces derniers sont confondus et donc identiques. Cette condition n'a pas de rôle dans le cas d'un regroupement d'unités spatiales. Lorsque deux unités possèdent une proximité nulle, cela implique qu'elles entretiennent une forte interaction spatiale et donc qu'elles participent à un même groupe. Enfin, la proximité calculée ne possède pas nécessairement la propriété d'inégalité triangulaire qui stipule qu'une distance entre i et $\mathrm{j}$ est toujours inférieure ou égale à la somme des distances entre ces deux lieux et un troisième. Selon la vérification ou non de cette dernière contrainte par le tableau des 
proximités, divers critères de regroupement sont envisageables pour effectuer la classification.

\section{Critère de regroupement des unités spatiales}

23 La règle fondamentale à respecter consiste à choisir un critère correspondant à la distance considérée. La proximité calculée précédemment permet d'envisager deux démarches en fonction de ses propriétés. Lorsqu'elle est euclidienne, les critères ${ }^{1}$ de regroupements contenant des références au cadre euclidien sont utilisables, comme celui du moment centré d'ordre deux d'une partition. Dans le cas contraire, des critères plus généraux entrent en ligne de compte, telle la distance moyenne.

\section{Discontinuité d'intensité de relation et identification du système urbain quotidien}

24 La classification des individus fournit un arbre hiérarchique de regroupement montrant leur degré de dissemblance. Les discontinuités marquantes permettent d'identifier des sous-ensembles de lieux entretenant des relations privilégiées. Une démarche possible consiste à prendre les degrés de dissemblance à partir du dernier groupe formé jusqu'à ce que la succession ordonnée des degrés ne fasse plus apparaitre de rupture importante entre deux degrés successifs. La troncature de l'arbre hiérarchique fournit alors un ensemble de $\mathrm{n}$ groupes qui sont rassemblés en fonction de l'appartenance de certaines de leurs communes à la même unité urbaine. On aboutit ainsi à la constitution du système urbain quotidien. Les différentes étapes de cette constitution sont résumées sur la figure 2 et illustrées dans les paragraphes suivants à partir de l'exemple du système strasbourgeois. 
Figure 2 : Etapes d'identification du système urbain quotidien

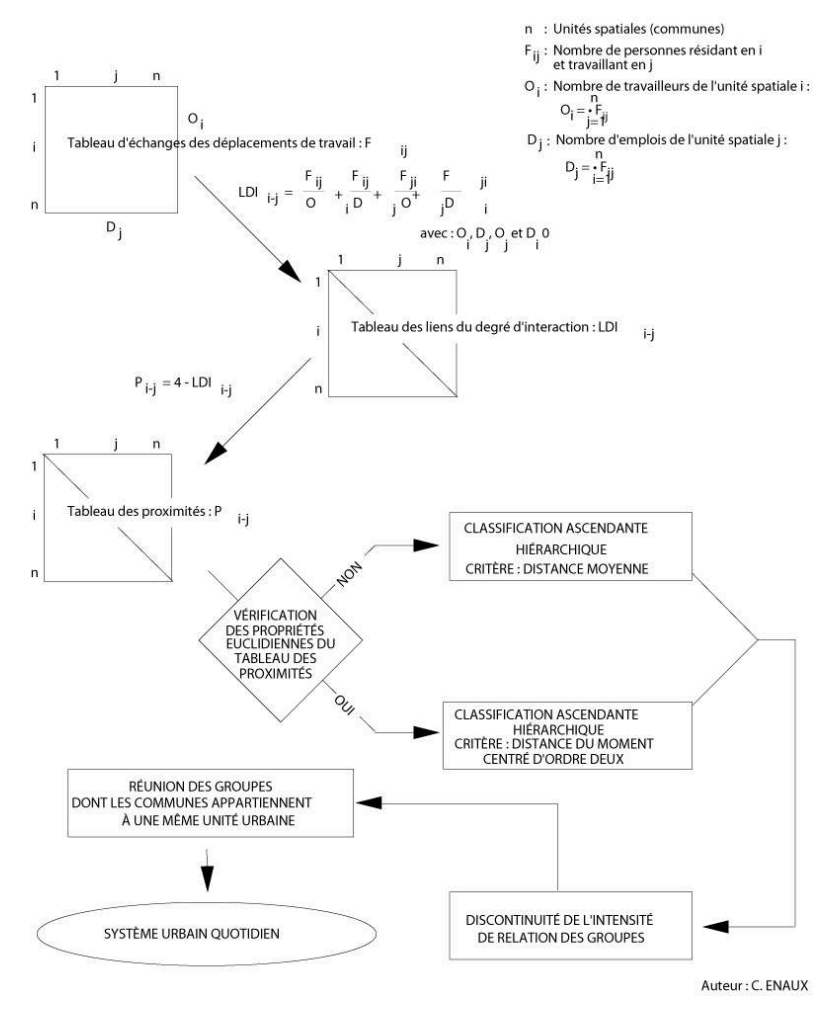

\section{Exemple du système urbain quotidien strasbourgeois}

Nous disposions au départ du tableau d'échanges de flux de déplacements de travail des communes du département du Bas-Rhin au recensement de $1990^{2}$. Ce tableau a subi deux transformations, la première dans le but de calculer les liens du degré d'interaction et la seconde fournissant le tableau des proximités entre les communes. Sur ce dernier, la propriété d'inégalité triangulaire a été testée et le résultat indique que tous les triplets de lieux sont espacés de telle façon qu'une distance entre deux lieux est toujours inférieure ou égale à celle passant par un troisième lieu. Ce respect de l'inégalité triangulaire s'explique dans notre cas par la grande dispersion des flux des communes. Le critère du moment centré d'ordre deux a donc pu être employé dans le cadre d'une classification ascendante hiérarchique ${ }^{3}$.

Les degrés de dissemblance des groupes constituant l'arbre hiérarchique sont représentés sous la forme d'un graphique (fig. 3a) dont l'axe des abscisses comporte les numéros des différents groupes et l'axe des ordonnées le degré de dissemblance de ceux-ci. Ce graphique montre que la courbe possède trois parties bien distinctes. La première est formée des groupes dont le numéro est inférieur à 600 . Elle indique une rapide augmentation de la dissemblance des groupes qui traduit la forte dispersion des flux de déplacements de travail des communes. En effet, ces dernières entretiennent des liens avec un nombre plus élevé de communes que la dimension restreinte des ensembles ne peut saisir à ce stade du regroupement. Lorsque les groupes comportent plus d'individus, l'augmentation du degré de dissemblance est beaucoup plus lente. Elle montre que l'on entre dans une phase où chaque regroupement produit une 
différenciation interne des agrégats moins accentuée et les communes d'un groupe forment alors un sous-ensemble d'individus ayant une prépondérance de liens entre eux. La dernière partie enfin, composée des groupes ayant des numéros supérieurs à 950 , fait apparaître un accroissement quasi-exponentiel des degrés de dissemblance. La différenciation interne des groupes est de plus en plus grande puisque l'on réunit des sous-ensembles dont l'intensité des liens diminue fortement.

Figure 3 : Degrés de dissemblance de la classification des communes du département du Bas-Rhin
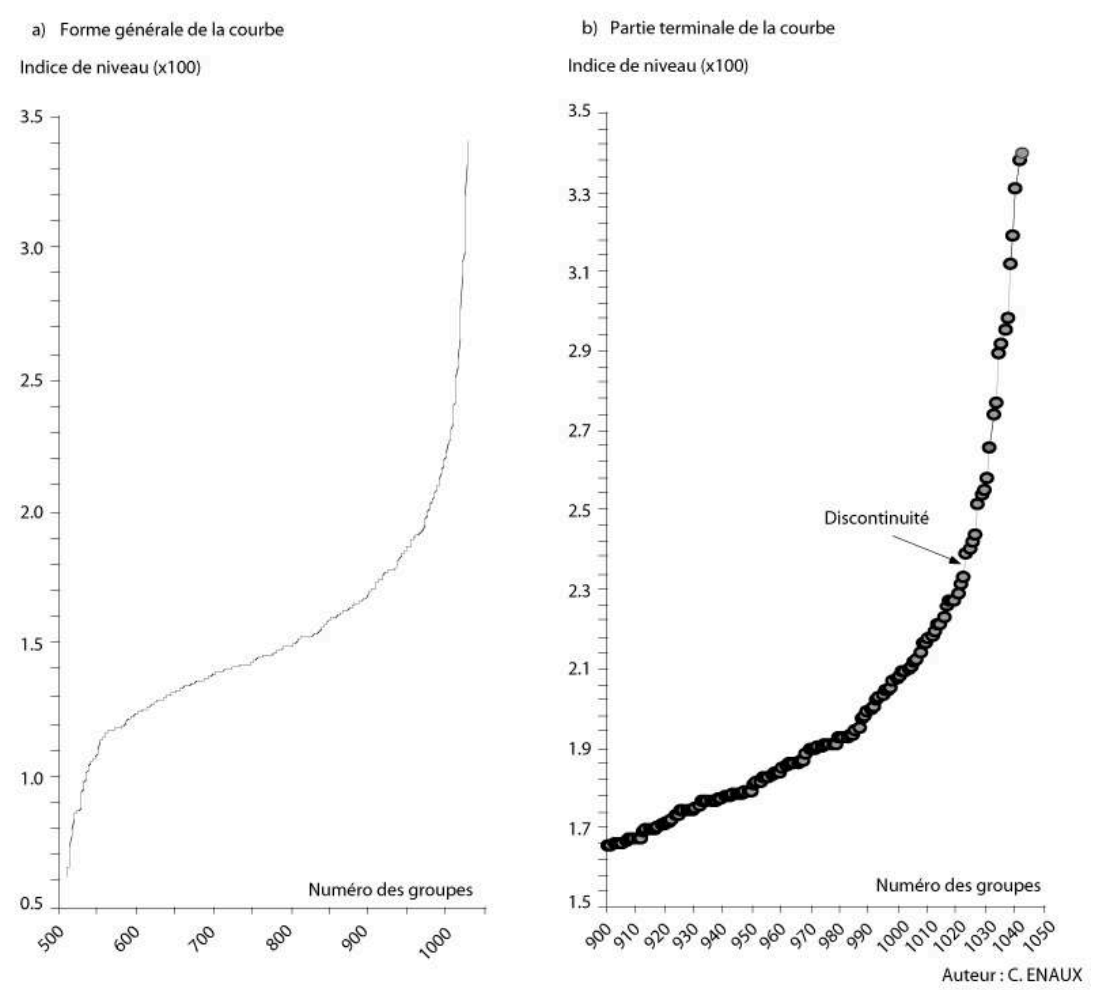

Un zoom sur cette partie terminale de la courbe (fig. $3 b$ ) permet de repérer les discontinuités majeures entre les degrés de dissemblance. On observe une discontinuité des indices de niveau plus ou moins importante jusqu'au groupe 1023. Ensuite, les degrés de dissemblance ne font plus apparaître de rupture majeure. C'est donc juste avant la constitution du groupe 1024 que nous avons effectué la coupure dans le dendrogramme, fournissant ainsi vingt et un groupes qui sont assemblés selon que les communes qui les composent appartiennent ou non à la même unité urbaine.

La figure 4 présente les ensembles de communes composant le système urbain quotidien de l'agglomération strasbourgeoise ${ }^{4}$. Le groupe 1011 constitue le cœur de ce système urbain et comporte la plupart des principales communes de l'unité urbaine. Ce dernier entretient des relations privilégiées avec l'ensemble 1022, des communes dont la partie principale est située dans la région du Kochersberg, un espace toujours agricole parsemé de petits villages et de bourgs. La proximité de ces deux groupes est liée à l'absence de pôles secondaires d'emplois dans le Kochersberg. Ainsi la majorité de la population de ce secteur vient-elle exercer son activité professionnelle dans le grand pôle strasbourgeois. Mais la périurbanisation et la rurbanisation clairement identifié par P. Limouzin (1988) dans le Kochersberg, contribuent également à cette proximité. 
De nombreux actifs de l'agglomération strasbourgeoise sont venus s'installer en bordure de cette dernière tout en conservant leur emploi dans l'agglomération.

Figure 4 : Système urbain quotidien strasbourgeois en 1990

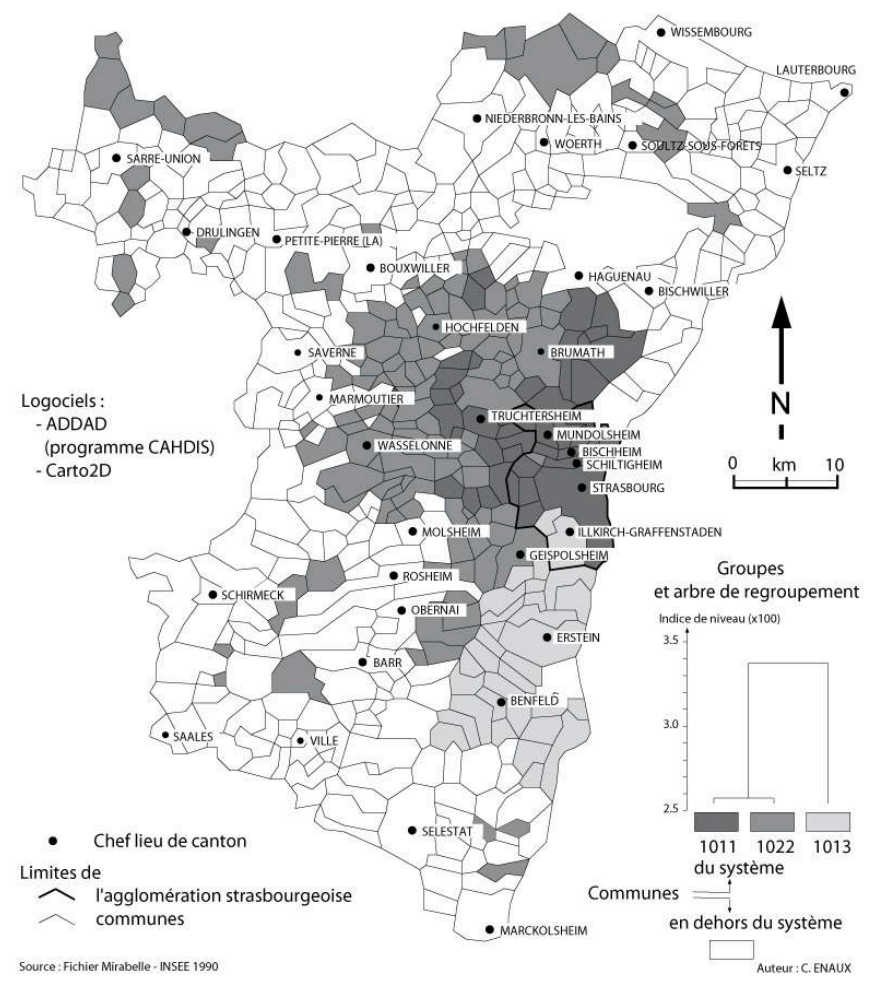

Ce système urbain quotidien comporte un troisième groupe de communes (groupe 1013) qui possède cependant des liens beaucoup plus faibles avec les deux ensembles précédents. Il contient la commune d'Illkirch-Graffenstaden, un des principaux pôle d'emplois du département du Bas-Rhin, caractérisé par ses nombreuses zones d'activités, mais dont la population active provient principalement des communes situées le long d'un axe routier et ferroviaire important, passant par Erstein et Benfeld. On constate ainsi une organisation sectorielle du système urbain quotidien strasbourgeois, avec une partie principale constituée des groupes 1011 et 1022 et une autre partie, secondaire, formée des communes du groupe 1013. Ce phénomène n'est en rien surprenant puisque le lien du degré d'interaction constitue des groupes de solidarité économique et résidentielle.

Une double logique spatiale se superpose à cette organisation sectorielle du système urbain quotidien. On distingue un ensemble principal de communes jointives situées sur le pourtour de l'agglomération strasbourgeoise. Alors que le regroupement s'effectue uniquement selon le degré d'interaction des communes, le système urbain quotidien est majoritairement composé de communes contiguës. Ce phénomène traduit l'importance encore actuelle, malgré le développement des facilités de déplacement, de la proximité géographique des communes. Cependant, le système urbain exprime également sur ses marges, une logique d'éclatement spatial. Cette tendance est observable à travers les divers îlots de communes appartenant au groupe 1022. Il est clair que la masse d'emplois du système urbain strasbourgeois exerce une attraction sur la population de communes parfois très éloignées. Mais on peut également se 
demander si certaines d'entre elles ont été intégrées dans le système par absence du tableau d'échanges de déplacements de travail des communes avec lesquelles elles ont des relations privilégiées. Après vérification, il apparait que les communes situées à l'ouest de La Petite Pierre présentent de fortes relations avec des communes absentes du tableau, comme celles de l'agglomération de Sarreguemines, ou de Sarrebourg, ou encore de Phalsbourg. Il en va de même avec les communes situées au Nord de Haguenau, qui ont des liens majoritairement avec l'Allemagne, probablement avec la grande agglomération de Karlsruhe (le fichier fournit par l'I.N.S.E.E. ne précise pas les communes de destination situées hors des frontières nationales). On peut donc proposer de les soustraire du système urbain quotidien strasbourgeois.

31 La délimitation d'un système urbain quotidien, comme elle a été envisagée précédemment, induit une démarche qui permet de définir des systèmes spatiaux fondés sur des éléments de cohérence interne. Cette démarche aboutit à des ensembles spatiaux dont la continuité n'est pas nécessairement une caractéristique et dont l'hétérogénéité s'oppose à des secteurs géographiques délimités traditionnellement à partir du critère d'homogénéité. La démarche mise en œuvre possède une portée d'ordre général pour le géographe à la recherche d'une zone d'étude d'un type de mouvements à travers l'espace géographique. En effet, il suffit de déterminer un ensemble d'unités spatiales entretenant des relations privilégiées à partir d'un tableau de mouvements exhaustif et suffisamment large, pour faire apparaître le système spatial fondé sur ces mouvements. Les différentes étapes nécessaires à son identification se résument alors :

- à une transformation du tableau d'échanges en un tableau de liens, afin de mettre en lumière la structure des relations et non celle liée à la « masse » des échanges ;

- à une modification du tableau de liens en tableau de proximités ;

- à la vérification des propriétés du tableau des proximités ;

- à la réalisation d'une classification ascendante hiérarchique à l'aide d'un critère de regroupement des individus adapté aux propriétés du tableau ;

- à l'identification du seuil de rupture des degrés de dissemblance des différents groupes d'unités spatiales.

L'identification de systèmes spatiaux conduit donc à la mise en lumière d'objets véritablement géographiques. L'espace ne possède pas son rôle habituel de cadre spatial neutre, mais intervient directement comme un facteur constitutif des objets à l'instar des processus sociaux. Loin d'être négligeable, une telle approche fournit aux géographes des entités dont l'étude possède alors une certaine cohérence par rapport au champ scientifique de la discipline.

\section{BIBLIOGRAPHIE}

Berry B.J.L. et Horton F. E. (1970). - Geographic perspective on Urban Systems. Prentice-Hall Inc, $564 \mathrm{p}$. 
Claval P. (1984). - Géographie humaine et économique contemporaine. Collection Fondamental, P.U.F., $442 \mathrm{p}$.

Le Jeannic T. (1996). - La délimitation des nouvelles aires urbaines. In : Données Urbaines, Collection Anthropos - Villes, Economica, pp. 165-172.

Limouzin P et al. (1988). - Les mutations spatiales dans l'agglomération de Strasbourg et sa périphérie. Collection Publications de la Maison des Sciences de l'homme de Strasbourg, $\mathrm{n}^{\circ} 4$. Association des publications près les universités de Strasbourg, $250 \mathrm{p}$.

Marchetti C. (1991). - Voyager dans le temps : considérations pour une meilleure exploitation de la liaison fixe. In : Futuribles, $\mathrm{n}^{\circ}$ juillet-août, pp. 19-29.

Masser I. et Brown P.J.B. (1975). - Hierarchical aggregation procedures for interaction data. In : Environnement and Planning A, vol. $n^{\circ}$ 7, pp. 509-523.

Orfeuil J.-P. et al. (1989). - Un milliard de déplacements par semaine : la mobilité des Français. La Documentation Française, 293 p.

Pumain D., Saint-Julien T., Cattan N. et Rozenblat C. (1990). - Le concept statistique de la ville en Europe. Rapport remis à l'office Statistique des Communautés Européennes, 88 p.

Ward J.H. (1963). - Hierarchical grouping to optimaze an objective fonction. In : Journal of the American Statistical Association, vol. $\mathrm{n}^{\circ}$ 58, pp. 236-244.

\section{NOTES}

1. Les critères de regroupement ainsi que leurs avantages/inconvénients respectifs sont présentés notamment dans l'ouvrage de : SANDERS L. (1989). - L'analyse des données appliquée à la géographie. Collection ALILADE, G.I.P. RECLUS, 268 p.; ou dans celui de: JAMBU M. (1989). Exploration informatique et statistique des données. Collection Technique et Scientifique des Télécommunications, Dunod, 505 p.

2. Le tableau global comporte un total de 522 communes, plus une ligne et une colonne spéciales, tenant compte respectivement des flux en provenance et à destination de l'extérieur du département.

3. Elle a été réalisée avec le programme CAHDIS du logiciel d'analyse de données ADDAD.

4. Les communes restant en dehors du système strasbourgeois participent à d'autres systèmes quotidiens non représentés sur la figure.

\section{RÉSUMÉS}

Une délimitation du système urbain quotidien est proposée à partir d'une discussion sur les logiques possibles et les approches envisageables. Le principe méthodologique retenu est présenté en détail et illustré à l'aide de l'exemple du système urbain quotidien strasbourgeois. La portée générale de la démarche est mise en avant pour déterminer des portions cohérentes d'espace géographique permettant notamment d'étudier des phénomènes de relations entre les lieux. 
A delimitation of the daily urban system is discussed in this paper, based on a presentation of logical possibilities and different imaginable approaches. The adopted methodology is fully detailed and illustrated using the exemple of the daily urban system of Strasbourg. The possibility of generalisation of the method is stressed here, in order to determine coherent parts of geographical space which especially allow the study of relations among different places.

Es wird eine Abgrenzung des «täglichen Stadtsystems » vorgeschlagen, ausgehend von einer Diskussion über mögliche Logik und erfassbare methodische Zugänge. Das hier angewandte methodologische Prinzip wird im Detail dargestellt und mit Hilfe des Beispiels des Stadtsystems von Strassburg veranschaulicht. Hervorgehoben wird die allgemeine Bedeutung des Verfahrens zur Bestimmung der kohärenten Teile des geographischen Raums ; dies gestattet namentlich, die Phänomene der Beziehungen zwischen den Orten zu studieren.

\section{INDEX}

Mots-clés : degré d'interaction, déplacements de travail, système urbain quotidien Schlüsselwörter : Berufspendelwanderung, Interaktiongrad, tägliches Stadtsystem Keywords : daily urban system, degree of interaction, journeys-to-work

\section{AUTEUR}

\section{CHRISTOPHE ENAUX}

Laboratoire «Image et Ville » - UPRES-A 7011 CNRS - Université Louis-Pasteur - 3, rue de l'Argonne 67000 Strasbourg 